\title{
Pseudomyxoma peritonei: a rare disease and a disease model in peritoneal surface oncology
}

\author{
Santiago González-Moreno
}

$\mathrm{P}^{\mathrm{e}}$ ritoneal dissemination of abdominal and pelvic-based malignancies has attracted the clinical and scientific interest of surgical oncologists in the last two decades. Unprecedented survival results have been reported by the knowledgeable use of cytoreductive surgery combined with perioperative intraperitoneal chemotherapy and systemic chemotherapy in patients suffering from peritoneal carcinomatosis of various origins [1]. As more and more sound scientific data in this direction are produced and published, peritoneal disease treatment centres have been established in almost every country around the world. Peritoneal surface oncology is a reality.

Not every patient with peritoneal carcinomatosis or a primary peritoneal neoplasm is a candidate for a radical combined treatment. Careful patient selection by a dedicated multidisciplinary team is the first requirement for success [2]. The whole justification for a radical locoregional treatment lies in the presumed absence of tumour dissemination beyond the peritoneal cavity, a fact that is frequently observed at a given time point in the natural history of gastrointestinal or gynaecological cancers. And that is the moment at which patients may be considered for a curative-intent treatment.

Pseudomyxoma peritonei (PMP) is a fascinating disease characterised by mucinous peritoneal implants and/ or mucinous ascites, originating in the vast majority of cases from a ruptured mucinous appendiceal neoplasm. It is a rare disease, with a reported incidence of one case per million per year. Therefore, it is unlikely that colleagues working in population-based healthcare centres will gain any but anecdotal experience on its diagnosis and treatment. Subsequently, an educational effort to avoid feeding

S. González-Moreno ( $₫)$

Department of Surgical Oncology

Peritoneal Surface Oncology Program

Centro Oncológico MD Anderson International España

C/ Arturo Soria, 270

ES-28033 Madrid, Spain

e-mail: sgonzalez@mdanderson.es

misconceptions on the disease and errors in its appropriate management is required [3].

It is intriguing to realise how such an uncommon disease has produced such a large number of studies and publications in the biomedical literature in the recent past that have led to a precise pathological characterisation that unveils its diverse nature, a clear description of its prognostic factors and a consensus on its clinical management, with the reporting of many single-centre clinical patient series. The answer lies in the fact that PMP constitutes the perfect example of a peritoneal neoplastic disease that will only exceptionally metastasise outside of the peritoneal cavity. This peculiar biological behaviour and its slow progress over time make PMP a paradigm, a disease model in peritoneal surface oncology, as it exemplifies the disease status that is amenable to radical loco-regional therapy. Furthermore, PMP also boasts the best treatment results that can be obtained by its application in any peritoneal malignant disease. In fact it is the only approved indication for cytoreductive surgery and hyperthermic intraperitoneal chemotherapy (HIPEC) by the National Health Service of the United Kingdom and an unquestionable indication for this combined, often criticised, treatment option [4]. The scientific justification for its use as the standard of care in PMP will never come from randomised clinical trials, but it is well based in the results of numerous phase II studies, some of them including hundreds of patients [5], that compare very favourably with those of historical controls treated with serial debulking [6]. An ambitious international PMP registry project is underway, assembled and coordinated from the Peritoneal Surface Malignancy Program led by Professor Morris at St. George's Hospital in Sydney, Australia, which will certainly make another invaluable contribution to the knowledge of this disease and its treatment.

Early in its short history, the Spanish Peritoneal Surgical Oncology Group (GECOP) designed and agreed on a national PMP treatment protocol that combines cytoreductive surgery and HIPEC, adding adjuvant systemic chemotherapy only when indicated by unfavourable histopathological criteria [7]. This issue of Clinical and Translational Oncology includes the experience in the management of PMP from one of GECOP's national referral centres for 
peritoneal surface malignancies [8]. Arjona-Sánchez and colleagues contribute in this way to raise awareness of the need for centralised, specialised management and add to the reported phase II efforts on this disease. Their series features a majority of cases $(80 \%)$ of the unfavourable mucinous peritoneal carcinomatosis or hybrid histopathological subtypes, but their results still fall within the expected outcomes, as published by other groups. Their accumulated vast experience with this complex procedure in other malignant peritoneal diseases allows for a very high rate $(93 \%)$ of complete cytoreductions (the primary goal of this treatment) at an affordable treatment-related morbidity and mortality, for which they should be congratulated.
PMP has certainly played a key role in the development of cytoreductive surgery combined with perioperative intraperitoneal chemotherapy for peritoneal surface malignancies, a contribution for which Paul H. Sugarbaker should be undoubtedly credited. Lessons learnt from the radical treatment of this disease have been successfully applied to selected cases of peritoneal carcinomatosis of colorectal or gastric origin, peritoneal mesothelioma or stage III ovarian cancer. These lessons include, among many others, early detection and treatment, multidisciplinary management and centralisation in expert treatment centres. It may be stated that never before has a rare disease contributed so much to the development of modern surgical oncology.

\section{References}

1. Glehen O, Gilly FN, Boutitie F et al (2010) Toward curative treatment of peritoneal carcinomatosis from nonovarian origin by cytoreductive surgery combined with perioperative intraperitoneal chemotherapy: a multi-institutional study of 1,290 patients. Cancer 116:5608-5618

2. González-Moreno S, Ortega-Pérez G, González Bayón L (2009) Indications and patient selection for cytoreductive surgery and perioperative intraperitoneal chemotherapy. J Surg Oncol 100:287-292
3. González-Moreno S, Bretcha Boix P, Rufián Peña $S$, Muñoz Casares FC, in representation of GECOP (2011) Important concepts on the treatment of appendiceal mucinous neoplasms and "pseudomyxoma peritonei". Cir Esp 89:200-201

4. González-Moreno S (2006) Surgical management of peritoneal carcinomatosis: from clinical research to standard of care. Clin Transl Oncol 8: 545-546

5. Sugarbaker PH (2009) Epithelial appendiceal neoplasms. Cancer J 15:225-235

6. Sugarbaker PH (2006) New standard of care for appendiceal epithelial neoplasms and pseudomyxoma peritonei syndrome? Lancet Oncol 7:69-76
7. Torres Melero J, Fernández A, Reche $\mathrm{P}$ et al (2009) Propuesta de un Protocolo Nacional para el Pseudomixoma peritoneal (carcinomatosis mucinosa) mediante técnicas de citorreducción y quimioterapia intraperitoneal perioperatoria. Abstract Book, poster 275, XII Congreso Nacional de la Sociedad Española de Oncología Médica (SEOM). Barcelona, 21-23 October

8. Arjona-Sánchez A, Muñoz-Casares FC, RufiánPeña S et al (2011) Pseudomyxoma peritonei treated by cytoreductive surgery and hyperthermic intraperitoneal chemotherapy: results from a single center. Clin Transl Oncol 13:261-267 